\title{
P. Goldberg et R. I. Macphail, Practical and theoretical geoarchaeology
}

Jean-Laurent Monnier

\section{(2) OpenEdition \\ Journals}

Édition électronique

URL : http://journals.openedition.org/rao/517

DOI : $10.4000 /$ rao. 517

ISBN : 978-2-7535-1606-9

ISSN : $1775-3732$

\section{Éditeur}

Presses universitaires de Rennes

\section{Édition imprimée}

Date de publication : 30 décembre 2006

Pagination : 227

ISBN : 978-2-7535-0574-2

ISSN : 0767-709X

\section{Référence électronique}

Jean-Laurent Monnier, « P. Goldberg et R. I. Macphail, Practical and theoretical geoarchaeology », Revue archéologique de l'Ouest [En ligne], 23 | 2006, mis en ligne le 30 décembre 2008, consulté le 03 décembre 2020. URL : http://journals.openedition.org/rao/517 ; DOI : https://doi.org/10.4000/rao.517 
Goldberg, P. et MaCphaIL, R. I., 2006 - Practical and theoretical geoarchaeology, London, Blackwell Publishing, 455 p. (ISBN 0-632-06044-1).

Paul Goldberg et Richard Macphail sont respectivement Professeur au Département d'Archéologie de l'Université de Boston et Chercheur à l'Institut d'Archéologie du Collège universitaire de Londres. Ils ont une connaissance large et approfondie du sujet traité et collaborent depuis longtemps en France avec nos équipes et dans nos projets de recherche.

La Géoarchéologie comprend l'ensemble des études et analyses concernant les sédiments en rapport avec les gisements archéologiques. Elle vise donc à connaitre les conditions de mise en place des dépôts, leur évolution, et donc contribue fondamentalement à l'approche taphonomique des sites. Elle est en tout cela essentielle et incontournable. La géoarchéologie regroupe donc les observations et analyses sédimentologiques, géochimiques, stratigraphiques, paléopédologiques, micromorphologiques...

L'ouvrage comprend de nombreuses figures et tableaux, une riche bibliographie et des index. À noter que la bibliographie comporte des références d'articles en français. Question de coût de fabrication bien sûr, un cahier intérieur de huit pages regroupe 14 photographies en couleurs, que l'on aurait souhaitées plus nombreuses dans le texte. Ce dernier est structuré en trois parties : 1) la géoarchéologie à l'échelle régionale; 2 ) les approches géoarchéologiques nontraditionnelles; 3) les méthodes de terrain et de laboratoire, le rendu et l'interprétation des données. Ces trois parties regroupent des chapitres bien équilibrés.

Le premier chapitre traite des sédiments. Les bases élémentaires de leur description (stratification, limites granulométriques, forme des grains, structures sédimentaires) sont données, en tenant compte notamment des diverses pratiques. Le second chapitre traite de la stratigraphie. Les principes fondamentaux, les définitions des unités stratigraphiques, les notions de faciès et microfaciès sont donnés, en distinguant bien lithostratigraphie, pédostratigraphie et archéostratigraphie, sans oublier les corrélations, dont l'exemple de la Matrice de Harris. Le troisième chapitre est consacré aux sols, aux facteurs de leur genèse et aux processus pédologiques, à la description des horizons, à leur classification élémentaire incluant leurs capacités spécifiques à conserver les vestiges archéologiques. La question des systèmes hydrologiques est partagée en deux chapitres : pentes et dépôts de pente (ch. 4), rivières et lacs (ch. 5). Les données essentielles, à la fois au point de vue dynamique et observations de terrain sont fournies. Le sixième chapitre traite des dépôts éoliens et des environnements archéologiques associés. Le chapitre 7 concerne les milieux côtiers (environnements littoraux, dépôts) et le chapitre 8 traite des grottes et abris sous roche (formation, évolution, anthropisation). Regrettons seulement que les milieux périglaciaires soient curieusement quasi absents.

Les " approches géoarchéologiques non-traditionnelles " se placent à une autre échelle et mettent l'accent sur les occupations humaines, leurs dépôts et actions sur les sédiments. C'est toute l'originalité et l'intérêt de cet ouvrage. Le chapitre 9 traite de l'impact humain sur les paysages (déforestation, modifications des sols, mises en culture). Le texte en est particulièrement précis et pertinent. Les dépôts correspondant aux occupations humaines sont du plus grand intérêt pour les archéologues. Les concepts et les aspects des dépôts " culturels " sont traités dans le chapitre 10, complétés (ch. 11) par d'excellents exemples issus de la riche expérience de terrain des auteurs, tant en Europe, qu'au Proche-Orient et en Amérique du Nord. Le chapitre 12 est consacré à la géoarchéologie expérimentale qui vient étayer et éclairer les observations sur les gisements archéologiques. Les matériaux mis en œuvre par l'homme (matériaux de construction, métallurgie...) entrent également dans le champ de la géoarchéologie (ch. 13). Curieusement la contribution de la géoarchéologie aux investigations judiciaires (ch. 14), mais qui pourrait s'étendre à l'anthropologie funéraire, mérite d'être signalée.

La troisième partie présente et explique les méthodes employées sur le terrain et en laboratoire. D'abord les méthodes de terrain (ch. 15), traitées de façon très complète et pédagogiques, ensuite les techniques de laboratoire (ch. 16), également bien développées; regrettons toutefois que la granulométrie ne soit pas exprimée sous la forme, plus habituelle et explicite, des courbes cumulatives en coordonnées semi-logarithmiques. Le chapitre 17 , tout à fait pertinent et bienvenu, explique comment préparer un rapport ou une publication dans le domaine concerné. La conclusion (ch. 18) évoque brièvement l'avenir de la géoarchéologie. À noter que ce livre apparait relativement pionnier en Amérique du Nord où la géoarchéologie est encore peu comprise et pratiquée. Le mérite des auteurs en est encore plus grand.

Il s'agit d'un ouvrage remarquable, construit de façon pédagogique, bien rédigé par des spécialistes très compétents. Il s'adresse utilement à tous les archéologues, et en particulier aux praticiens de l'archéologie préventive, aux étudiants avancés comme aux chercheurs et à des amateurs éclairés. On ne peut donc que le recommander fortement.

Jean-Laurent MonNiER (CNRS, UMR 6566, Université de Rennes 1) 